\title{
Approximation Properties of New Modified Gamma Operators
}

\author{
Yun-Shun Wu $\mathbb{D},{ }^{1}$ Wen-Tao Cheng $\mathbb{D}^{2}$, Wei-Ping Zhou $\mathbb{D}^{2},{ }^{2}$ and Lun-Zhi Deng $\mathbb{D}^{1}$ \\ ${ }^{1}$ School of Mathematical Sciences, Guizhou Normal University, Guizhou, Guiyang 550001, China \\ ${ }^{2}$ School of Mathematics and Physics, Anqing Normal University, Anhui, Anqing 246133, China
}

Correspondence should be addressed to Wen-Tao Cheng; chengwentao_0517@163.com

Received 7 April 2021; Revised 25 April 2021; Accepted 28 April 2021; Published 15 May 2021

Academic Editor: Tuncer Acar

Copyright ( 2021 Yun-Shun Wu et al. This is an open access article distributed under the Creative Commons Attribution License, which permits unrestricted use, distribution, and reproduction in any medium, provided the original work is properly cited.

This paper is aimed at constructing new modified Gamma operators using the second central moment of the classic Gamma operators. And we will compute the first, second, fourth, and sixth order central moments by the moment computation formulas, and their quantitative properties are researched. Then, the global results are established in certain weighted spaces and the direct results including the Voronovskaya-type asymptotic formula, and point-wise estimates are investigated. Also, weighted approximation of these operators is discussed. Finally, the quantitative Voronovskaya-type asymptotic formula and Grüss Voronovskaya-type approximation are presented.

\section{Introduction}

Recently, Karsli et al. [1] constructed and estimated the rate of convergence for functions with derivatives of bounded variation on $\mathbb{R}_{+}:=(0, \infty)$ of new Gamma type operators preserving $z^{2}$ as (see also [2])

$$
\left(\Phi_{l} \lambda(t)\right)(z)=\frac{(2 l+3) ! z^{l+3}}{l !(l+2) !} \int_{0}^{\infty} \frac{t^{l}}{(z+t)^{2 l+4}} \lambda(t) \mathrm{d} t, z \in \mathbb{R}_{+}
$$

In [3], Karsli et al. used analysis methods to obtain the rate of point-wise convergence for the operators (1). In [4], Karsli and Özarslan obtained some direct local and global approximation results for the operators (1). In [5], İzgi studied some direct results in asymptotic approximation about the operators (1). In [6], Krech gave a note about the results of İzgi in [5] and obtained an error estimate for the operators (1). In [7], Krech gave direct approximation theorems for the operators (1) in certain weighted spaces. In [8], Cai and Zeng constructed $q$-Gamma operators and gave their approximation properties. In [9], Zhao et al. extended the works of Cai and Zeng and considered the stancu generalization of $q$-Gamma operators. Recently, Cheng et al. constructed $(p, q)$-Gamma operators using $(p, q)$-Beta function of the second kind and discussed their approximation properties in [10]. In [11], Zhou et al. extended the works of Cheng et al. in [10] and constructed $(p, q)$-Gamma-Stancu operators. There are many papers about the research and application of other Gamma-type operators, and we mention some of them [12-17].

In this paper, we construct new modified Gamma operators using the second central moment of the operators (1) as follows:

Definition 1. For $l=1,2, \cdots$ and $\lambda: \mathbb{R}_{+} \longrightarrow \mathbb{R}$, we construct new modified Gamma operators by

$$
\left(Y_{l} \lambda(t)\right)(z)=\int_{0}^{\infty} K_{l}(t, z) \lambda(t) \mathrm{d} t, z \in \mathbb{R}_{+},
$$

where

$$
K_{l}(t, z)=\frac{2^{l}(2 l+3) ! ! z^{l+1}}{l !} \frac{t^{l}(z-t)^{2}}{(z+t)^{2 l+4}}, t, z \in \mathbb{R}_{+} .
$$

The paper is organized as follows: In Section 1, we introduce the history of Gamma operators and construct new modified Gamma operators using the second central moment. In Section 2, we obtain the basic results by the moment computation formulas. And the first, second, 
fourth, and sixth order central moment computation formulas and limit equalities are also obtained. In Section 3, we establish the global approximation results for the operators (2) in certain weighted spaces. In Sections 4 and 5, we investigate the direct results including the Voronovskaya-type asymptotic formula and point-wise estimates in three different Lipschitz classes and discuss weighted approximation. In Section 6, we present a quantitative Voronovskaya-type asymptotic formula and a Grüss Voronovskaya-type approximation (for the quantitative Voronovskaya type theorem and Grüss-Voronovskaya theorem for the other operators, see also [18-24]).

\section{Basic Results}

In this section, we present certain auxiliary results which will be used to prove our main theorems for the operators (2).

Lemma 2 (see [1]). For any $l \in \mathbb{N}_{+}, p=0,1,2, \cdots, l+2$, we have

$$
\varphi_{l}(p):=\left(\Phi_{l} t^{p}\right)(z)=\frac{(l+p) !(l+2-p) !}{l !(l+2) !} z^{p}, z \in \mathbb{R}_{+}
$$

Lemma 3. If we define $\phi_{l}(p):=\left(Y_{l} t^{p}\right)(z)$, then there holds the following relation

$$
\frac{2 z^{2}}{l+2} \phi_{l}(p)=\varphi_{l}(p+2)-2 \varphi_{l}(p+1) z+\varphi_{l}(p) z^{2},
$$

where $p=0,1, \cdots, l, z \in \mathbb{R}_{+}$.

Then, the following lemma can be obtained immediately:

Lemma 4. For any $l \in \mathbb{N}_{+}, z \in \mathbb{R}_{+}$, we have

$$
\begin{aligned}
\phi_{l}(0)= & 1 ; \phi_{l}(1)=\frac{l+3}{l} z ; \phi_{l}(2)=\frac{(l+2)(l+9)}{l(l-1)} z^{2}, \text { for } l>1 ; \\
\phi_{l}(3)= & \frac{(l+2)(l+3)(l+19)}{l(l-1)(l-2)} z^{3}, \text { for } l>2 ; \\
\phi_{l}(4)= & \frac{(l+2)(l+3)(l+4)(l+33)}{l(l-1)(l-2)(l-3)} z^{4}, \text { for } l>3 ; \\
\phi_{l}(p)= & \frac{(l-p) !(l+p) !\left(l+2 p^{2}+1\right)}{l !(l+1) !} z^{p}, \text { for } l \geq p ; \\
A_{l}(z):= & \left(Y_{l}(t-z)\right)(z)=\frac{3}{l} z ; \\
B_{l}(z):= & \left(Y_{l}(t-z)^{2}\right)(z)=\frac{6 l+24}{l(l-1)} z^{2}, \text { for } l>1 ; \\
& \left(Y_{l}(t-z)^{3}\right)(z)=\frac{90 l+230}{l(l-1)(l-2)} z^{3}, \text { for } l>2 ; \\
& \left(Y_{l}(t-z)^{4}\right)(z)=\frac{60\left(l^{2}+23 l+48\right)}{l(l-1)(l-2)(l-3)} z^{4}, \text { for } l>3 ;
\end{aligned}
$$

$$
\begin{gathered}
\left(Y_{l}(t-z)^{6}\right)(z)=\frac{840\left(l^{3}+69 l^{2}+506 l+768\right)}{l(l-1)(l-2)(l-3)(l-4)(l-5)} z^{6}, \text { for } l>5 \\
\lim _{l \longrightarrow \infty} A_{l}(z)=3 z \\
\quad \lim _{l \longrightarrow \infty} B_{l}(z)=6 z^{2} ; \\
\lim _{l \longrightarrow \infty} l^{2}\left(Y_{l}(t-z)^{4}\right)(z)=60 z^{4} \\
\lim _{l \longrightarrow \infty} l^{3}\left(Y_{l}(t-z)^{6}\right)(z)=840 z^{6}
\end{gathered}
$$

By the classical Korovkin theorem, we easily obtain the following lemma:

Lemma 5. For all $\lambda \in C_{B}\left(\mathbb{R}_{+}\right)$and any finite interval $I \subset \mathbb{R}_{+}$, then the sequence $\left\{\left(Y_{l} \lambda(t)\right)(z)\right\}$ converges to $\lambda$ uniformly on $I$, where $C_{B}\left(\mathbb{R}_{+}\right)$denotes the set of all real-valued bounded and continuous functions defined on $\mathbb{R}_{+}$, endowed with the norm $\|\lambda\|=\sup _{z \in \mathbb{R}_{+}}|\lambda(z)|$.

\section{Global Results}

In this section, we establish some global results by using certain Lipschitz classes. We first recall some basic definitions. Let $r \in \mathbb{N}:=\{0,1,2, \cdots\}$ and define the weighted function $w_{r}$ as follows:

$$
w_{0}(z):=1 \text { and } w_{r}(z):=\frac{1}{1+z^{r}} \text { for } z \in \mathbb{R}_{+} \text {and } r \in \mathbb{N} \backslash\{0\}
$$

Meantime, we consider the following subspace $S_{r}\left(\mathbb{R}_{+}\right)$of $C\left(\mathbb{R}_{+}\right)$generated by $w_{r}$ :

$S_{r}\left(\mathbb{R}_{+}\right):=\left\{\lambda \in C\left(\mathbb{R}_{+}\right): w_{r} \lambda\right.$ is uniformly continuous and bounded on $\left.\mathbb{R}_{+}\right\}$

endowed with the norm $\|\lambda\|_{r}:=\sup _{z \in \mathbb{R}_{+}} w_{r}(z)|\lambda(z)|$ for $\lambda \in S_{r}\left(\mathbb{R}_{+}\right)$. For every $\lambda \in S_{r}\left(\mathbb{R}_{+}\right), \delta>0$, and $\alpha \in(0,2]$, the usual weighted modulus of continuity, the second-order weighted modulus of smoothness, and the corresponding Lipschitz classes are, respectively, defined as

$$
\begin{aligned}
\omega_{r}^{1}(\lambda ; \delta) & :=\sup \left\{w_{r}(z)|\lambda(y)-\lambda(z)|:|y-z| \leq \delta, y, z \in \mathbb{R}_{+}\right\} ; \\
\omega_{r}^{2}(\lambda ; \delta) & :=\sup _{t \in(0, \delta]}\|\lambda(z+2 t)-2 \lambda(z+t)+\lambda(z)\|_{r} ; \\
\operatorname{Lip}_{r}^{2} \alpha & :=\left\{\lambda \in S_{r}\left(\mathbb{R}_{+}\right): \omega_{r}^{2}(\lambda ; \delta)=O\left(\delta^{\alpha}\right) \text { as } \delta \longrightarrow 0^{+}\right\} .
\end{aligned}
$$

Theorem 6. Let $r \in\{0,1, \cdots, l\}$ be fixed. Then, there exists a positive constant $C_{r}$ such that 


$$
\left\|\left(Y_{l}\left(\frac{1}{w_{r}}\right)\right)\right\|_{r} \leq C_{r}
$$

Furthermore, for all $\lambda \in S_{r}\left(\mathbb{R}_{+}\right)$, we have

$$
\left\|Y_{l} \lambda\right\|_{r} \leq C_{r}\|\lambda\|_{r}
$$

Thus, $Y_{l}$ is a linear positive operator from $S_{r}\left(\mathbb{R}_{+}\right)$to $S_{r}\left(\mathbb{R}_{+}\right)$ for any $r \in\{0,1, \cdots, l\}$.

Proof. Inequality (22) is obvious for $r=0$. Assume that $l \geq r \geq 1$, using (6), we have

$$
\begin{aligned}
w_{r}(z)\left(Y_{l}\left(\frac{1}{w_{r}(t)}\right)\right)(z) & =w_{r}(z)\left(Y_{l}\left(1+t^{r}\right)\right)(z) \\
& =w_{r}(z)\left(Y_{l}(1)\right)(z)+w_{r}(z)\left(Y_{l}\left(t^{r}\right)\right)(z) \\
& =w_{r}(z)+w_{r}(z) \frac{(l-r) !(l+r) !\left(l+2 r^{2}+1\right)}{l !(l+1) !} z^{r} \\
& \leq C_{r} w_{r}(z)\left(1+z^{r}\right) \\
& =C_{r},
\end{aligned}
$$

where $C_{r}=\max \left\{1, \sup \left((l-r) !(l+r) !\left(l+2 r^{2}+1\right) / l !(l+1) !\right)\right.$ \} , and then we obtain (22). Moreover, for every $\lambda \in S_{r}\left(\mathbb{R}_{+}\right)$ and $z \in \mathbb{R}_{+}$, we have

$$
\begin{aligned}
w_{r}(z)\left|\left(Y_{l} \lambda(t)\right)(z)\right| & \leq w_{r}(z) \int_{0}^{\infty} K_{l}(t, z)|\lambda(t)| \mathrm{d} t \\
& =w_{r}(z) \int_{0}^{\infty} K_{l}(t, z)|\lambda(t)| \frac{w_{r}(t)}{w_{r}(t)} \mathrm{d} t \\
& \leq\|\lambda\|_{r} w_{r}(z)\left(Y_{l}\left(\frac{1}{w_{r}(t)}\right)\right)(z) \\
& \leq C_{r}\|\lambda\|_{r} .
\end{aligned}
$$

Taking the supremum over $z \in \mathbb{R}_{+}$, we obtain (23).

Theorem 7. For any fixed $r \in\{0,1, \cdots, l-2\}, l \geq 2$, there exists a positive constant $C_{r}$ such that

$$
w_{r}(z)\left(Y_{l}\left(\frac{(t-z)^{2}}{w_{r}(t)}\right)\right)(z) \leq C_{r} \frac{z^{2}}{l}
$$

Proof. The formula (11) implies (26) for $r=0$. If $r=1$, then we obtain

$$
\begin{aligned}
\left(Y_{l}\left((t-z)^{2}(1+t)\right)\right)(z) & =\left(Y_{l}\left((t-z)^{2}\right)\right)(z)+\left(Y_{l}\left((t-z)^{2} t\right)\right)(z) \\
& =\left(Y_{l}\left((t-z)^{3}\right)\right)(z)+(1+z)\left(Y_{l}\left((t-z)^{2}\right)\right)(z),
\end{aligned}
$$

which by (11) and (12) yield (26) for $r=1$. Assuming $l-2$ $\geq r \geq 2$ and using (11) and (6), we obtain

$$
\begin{aligned}
\left(Y_{l}\left(\frac{(t-z)^{2}}{w_{r}(t)}\right)\right)(z)= & \left(Y_{l}\left((t-z)^{2}\right)\right)(z)+\left(Y_{l}\left(t^{r+2}\right)\right)(z) \\
& -2 z\left(Y_{l}\left(t^{r+1}\right)\right)(z)+z^{2}\left(Y_{l}\left(t^{r}\right)\right)(z) \\
= & \frac{6 l+24}{l(l-1)} z^{2} \\
& +\frac{(l-r-2) !(l+r+2) !\left(l+2(r+2)^{2}+1\right)}{l !(l+1) !} z^{r+2} \\
& -2 \frac{(l-r-1) !(l+r+1) !\left(l+2(r+1)^{2}+1\right)}{l !(l+1) !} z^{r+2} \\
& +\frac{(l-r) !(l+r) !\left(l+2 r^{2}+1\right)}{l !(l+1) !} z^{r+2} \\
= & \frac{z^{2}}{l}\left\{\frac{6 l+24}{l-1}+\left\{( l + r + 1 ) ( l + r + 2 ) \left(l+2(r+2)^{2}\right.\right.\right. \\
& +1) 2(l-r-1)(l+r+1)\left(l+2(r+1)^{2}+1\right) \\
& \left.\left.+(l-r-1)(l-r)\left(l+2 r^{2}+1\right)\right\} \frac{(l-r-2) !(l+r) !}{(l-1) !(l+1) !} z^{r}\right\} \\
\leq & \frac{z^{2}}{l}\left(\frac{6 l+24}{l-1}+\frac{\left(6 l^{2}+C_{r, 1} l+C_{r, 2}\right)(l-r-2) !(l+r) !}{(l-1) !(l+1) !} z^{r}\right) \\
\leq & C_{r} \frac{z^{2}}{l}\left(1+z^{r}\right),
\end{aligned}
$$

where $C_{r, 1}$ and $C_{r, 1}$ are two constants only depending on $r$. This completes the proof.

Now, for $r \in\{0,1, \cdots, l\}$, we consider the two spaces $S_{r}^{1}\left(\mathbb{R}_{+}\right):=\left\{\lambda \in S_{r}\left(\mathbb{R}_{+}\right): \lambda^{\prime} \in S_{r}\left(\mathbb{R}_{+}\right)\right\}$and $S_{r}^{2}\left(\mathbb{R}_{+}\right):=\left\{\lambda \in S_{r}(\right.$ $\left.\left.\mathbb{R}_{+}\right): \lambda^{\prime \prime} \in S_{r}\left(\mathbb{R}_{+}\right)\right\}$, and we have the three following theorems:

Theorem 8. For any fixed $r$, if $\lambda \in S_{r}^{1}\left(\mathbb{R}_{+}\right)$, there exists a positive constant $C_{r}$ such that

$$
w_{r}(z)\left|\left(Y_{l} \lambda(t)\right)(z)-\lambda(z)\right| \leq C_{r}\left\|\lambda^{\prime}\right\|_{r} \frac{z}{\sqrt{l}}
$$

for all $z \in \mathbb{R}_{+}$and $l \geq r+2$.

Proof. Let $z \in \mathbb{R}_{+}$. By $\lambda(t)-\lambda(z)=\int_{t}^{z} \lambda^{\prime}(u) \mathrm{d} u, t \in \mathbb{R}_{+}$, Lemma (11), and the linearity of $Y_{l}$, we obtain

$$
\left(Y_{l} \lambda(t)\right)(z)-\lambda(z)=\left(Y_{l} \int_{z}^{t} \lambda^{\prime}(u) \mathrm{d} u\right)(z)
$$

Using

$$
\left|\int_{t}^{z} \lambda^{\prime}(u) \mathrm{d} u\right| \leq\left\|\lambda^{\prime}\right\|\left\|_{r}\left|\int_{z}^{t} \frac{1}{w_{r}(u)} \mathrm{d} u\right| \leq\right\| \lambda^{\prime} \|_{r}\left(\frac{1}{w_{r}(t)}+\frac{1}{w_{r}(z)}\right)|t-z| .
$$

Hence,

$$
\begin{aligned}
& w_{r}(z)\left|\left(Y_{l} \lambda(t)\right)(z)-\lambda(z)\right| \leq\left\|\lambda^{\prime}\right\|_{r} \\
& \quad \cdot\left(\left(Y_{l}|t-z|\right)(z)+w_{r}(z)\left(Y_{l}\left(\frac{|t-z|}{w_{r}(t)}\right)\right)(z)\right) .
\end{aligned}
$$


Applying the well-known Cauchy-Schwarz inequality, we can obtain

$$
\begin{aligned}
\left(Y_{l}|t-z|\right)(z) & \leq \sqrt{\left(Y_{l}(t-z)^{2}\right)(z)}, \\
\left(Y_{l}\left(\frac{|t-z|}{w_{r}(t)}\right)\right)(z) & \leq \sqrt{\left(Y_{l}\left(\frac{1}{w_{r}(t)}\right)\right)(z)} \sqrt{\left(Y_{l}\left(\frac{(t-z)^{2}}{w_{r}(t)}\right)\right)(z) .}
\end{aligned}
$$

Combining (22) and (26), we can get the required result.

Theorem 9. For any fixed $r$, if $\lambda \in S_{r}^{l}\left(\mathbb{R}_{+}\right)$, then there exists a positive constant $C_{r}$ such that

$$
w_{r}(z)\left|\left(Y_{l} \lambda(t)\right)(z)-\lambda(z)\right| \leq C_{r} \omega_{r}^{l}\left(\lambda ; \frac{z}{\sqrt{l}}\right)
$$

for all $z \in \mathbb{R}_{+}$and $l \geq r+2$.

Proof. Let $z \in \mathbb{R}_{+}$. We denote the Steklov means of $\lambda$ by $\lambda_{s}$, $s \in \mathbb{R}_{+}$:

$$
\lambda_{s}(z)=\frac{1}{s} \int_{0}^{s} \lambda(u+z) \mathrm{d} u, z, s \in \mathbb{R}_{+}
$$

It is obvious that

$$
\begin{aligned}
\lambda_{s}(z)-\lambda(z) & =\frac{1}{s} \int_{0}^{s}(\lambda(u+z)-\lambda(z)) \mathrm{d} u, \\
\lambda_{s}^{\prime}(z) & =\frac{1}{s}(\lambda(z+s)-\lambda(z))
\end{aligned}
$$

for $z, s \in \mathbb{R}_{+}$. Hence, if $\lambda \in S_{r}^{1}\left(\mathbb{R}_{+}\right)$, then $\lambda_{s} \in S_{r}^{2}\left(\mathbb{R}_{+}\right)$for every fixed $s \in \mathbb{R}_{+}$. Furthermore, we have

$$
\left\|\lambda_{s}-\lambda\right\|_{r} \leq \omega_{r}^{1}(\lambda ; s),\left\|\lambda_{s}^{\prime}\right\|_{r} \leq \frac{1}{s} \omega_{r}^{1}(\lambda ; s)
$$

By

$$
\begin{gathered}
w_{r}(z)\left|\left(Y_{l} \lambda(t)\right)(z)-\lambda(z)\right| \leq w_{r}(z)\left|\left(Y_{l}\left(\lambda(t)-\lambda_{s}(t)\right)\right)(z)\right| \\
+w_{r}(z)\left|\left(Y_{l} \lambda_{s}(t)\right)(z)-\lambda_{s}(z)\right|+w_{r}(z)\left|\lambda(z)-\lambda_{s}(z)\right|
\end{gathered}
$$

Using (23) and (37), we have

$$
w_{r}(z)\left|\left(Y_{l}\left(\lambda(t)-\lambda_{s}(t)\right)\right)(z)\right| \leq C_{r}\left\|\lambda-\lambda_{s}\right\|_{r} \leq C_{r} \omega_{r}^{1}(\lambda ; s)
$$

for any $z, s \in \mathbb{R}_{+}$. From (29) and (37), we have

$$
w_{r}(z)\left|\left(Y_{l} \lambda_{s}(t)\right)(z)-\lambda_{s}(z)\right| \leq C_{r}\left\|\lambda_{s^{\prime}}\right\|_{r} \frac{z}{\sqrt{l}} \leq C_{r} \frac{1}{s} \omega_{r}^{1}(\lambda ; s) \frac{z}{\sqrt{l}} .
$$

By (37), we have

$$
w_{r}(z)\left|\lambda(z)-\lambda_{s}(z)\right| \leq\left\|\lambda-\lambda_{s}\right\|_{r} \leq \omega_{r}^{1}(\lambda ; s)
$$

for any $z, s \in \mathbb{R}_{+}$. Finally, we have

$$
w_{r}(z)\left|\left(Y_{l} \lambda(t)\right)(z)-\lambda(z)\right| \leq \omega_{r}^{1}(\lambda ; s)\left(C_{r}+\frac{1}{s} C_{r} \frac{z}{\sqrt{l}}+1\right)
$$

for any $z, s \in \mathbb{R}_{+}$. Choosing $s=z / \sqrt{l}$, the proof is proved.

Theorem 10. Defining a new operator,

$$
\left(Y_{l}^{*} \lambda(t)\right)(z)=\left(Y_{l} \lambda(t)\right)(z)-\lambda\left(z+A_{l}(z)\right)+\lambda(z) .
$$

For any fixed $r$, if $\lambda \in S_{r}^{2}\left(\mathbb{R}_{+}\right)$, then there exists a positive constant $C_{r}$ such that

$$
w_{r}(z)\left|\left(Y_{l}^{*} \lambda(t)\right)(z)-\lambda(z)\right| \leq C_{r}\left\|\lambda^{\prime \prime}\right\|_{r} \frac{z^{2}}{l}
$$

for all $z \in \mathbb{R}_{+}$and $l \geq r+2$.

Proof. Using Taylor's expansion, we have

$$
\lambda(t)-\lambda(z)=(t-z) \lambda^{\prime}(z)+\int_{z}^{t}(t-u) \lambda^{\prime \prime}(u) \mathrm{d} u, z, t \in \mathbb{R}_{+} .
$$

By $\left(Y_{l}^{*}(t-z)\right)(z)=0$ and $\left(Y_{l}^{*} 1\right)(z)=1$, we have

$$
\begin{aligned}
& \left|\left(Y_{l}^{*} \lambda(t)\right)(z)-\lambda(z)\right| \leq\left(Y_{l}^{*}(\lambda(t)-\lambda(z))\right)(z) \\
& \quad \leq\left(Y_{l}^{*}\left(\int_{z}^{t}(t-u) \lambda^{\prime \prime}(u) \mathrm{d} u\right)\right)(z) \mid\left(Y_{l}\left(\int_{z}^{t}(t-u) \lambda^{\prime \prime}(u) \mathrm{d} u\right)\right)(z) \\
& \quad-\int_{z}^{z+A_{l}(z)}\left(z+A_{l}(z)-u\right) \lambda^{\prime \prime}(u) \mathrm{d} u \mid .
\end{aligned}
$$

Since

$$
\begin{aligned}
& \left|\int_{z}^{t}(t-u) \lambda^{\prime \prime}(u)\right| \leq \frac{\left\|\lambda^{\prime \prime}\right\|_{r}(t-z)^{2}}{2}\left(\frac{1}{w_{r}(z)}+\frac{1}{w_{r}(t)}\right), \\
& \left|\int_{z}^{z+A_{l}(z)}\left(z+A_{l}(z)-u\right) \lambda^{\prime \prime}(u) \mathrm{d} u\right| \leq \frac{\left\|\lambda^{\prime \prime}\right\|_{r}}{2 w_{r}(z)}\left(A_{l}(z)\right)^{2},
\end{aligned}
$$


we have

$$
\begin{aligned}
& w_{r}(z)\left|\left(Y_{l}^{*} \lambda(t)\right)(z)-\lambda(z)\right| \leq \frac{\left\|\lambda^{\prime \prime}\right\|_{r}}{2} \\
& \cdot\left(B_{l}(z)+w_{r}(z)\left(Y_{l}\left(\frac{(t-z)^{2}}{w_{r}(t)}\right)\right)(z)\right) \\
& +\frac{\left\|\lambda^{\prime \prime}\right\|_{r}}{2}\left(A_{l}(z)\right)^{2} .
\end{aligned}
$$

Combining Lemma 4 and (26), we have

$$
w_{r}(z)\left|\left(Y_{l}^{*} \lambda(t)\right)(z)-\lambda(z)\right| \leq C_{r}\left\|\lambda^{\prime \prime}\right\|_{r} \frac{z^{2}}{l}
$$

for all $z \in \mathbb{R}_{+}$and $l \geq r+2$. The theorem is completed.

Theorem 11. For any fixed $r$, if $\lambda \in S_{r}^{2}\left(\mathbb{R}_{+}\right)$, then there exists a positive constant $C_{r}$ such that

$$
w_{r}(z)\left|\left(Y_{l} \lambda(t)\right)(z)-\lambda(z)\right| \leq C_{r} \omega_{r}^{2}\left(\lambda ; \frac{z}{\sqrt{l}}\right)+\omega_{r}^{1}\left(\lambda ; A_{l}(z)\right)
$$

for all $z \in \mathbb{R}_{+}$and $l \geq r+2$. In particular, if $\lambda \in \operatorname{Lip}_{r}^{2} \alpha$ for some $\alpha \in(0,2]$, then

$$
w_{r}(z)\left|\left(Y_{l} \lambda(t)\right)(z)-\lambda(z)\right| \leq C_{r}\left(\frac{z^{2}}{l}\right)^{\alpha / 2}+\omega_{r}^{1}\left(\lambda ; A_{l}(z)\right)
$$

holds.

Proof. Let $\lambda \in S_{r}\left(\mathbb{R}_{+}\right)$, and the Steklov means $\tilde{\lambda}_{s}(z)$ of the second order of $\lambda$ defined by

$$
\tilde{\lambda}_{s}(z)=\frac{4}{s^{2}} \int_{0}^{s / 2} \int_{0}^{s / 2}(2 \lambda(z+u+v)-\lambda(z+2 u+2 v)) \mathrm{d} u \mathrm{~d} v
$$

for $z, s \in \mathbb{R}_{+}$. By simple computation, we have

$$
\begin{aligned}
& \left\|\lambda-\tilde{\lambda}_{s}\right\|_{r} \leq \omega_{r}^{2}(\lambda ; s), \\
& \left\|\tilde{\lambda}_{s}^{\prime \prime}\right\|_{r} \leq \frac{9}{s^{2}} \omega_{r}^{2}(\lambda ; s) .
\end{aligned}
$$

Meantime, $\tilde{\lambda}_{s} \in S_{r}^{2}\left(\mathbb{R}_{+}\right)$while $\lambda \in S_{r}\left(\mathbb{R}_{+}\right)$. Using the following inequality,

$$
\begin{aligned}
\left|\left(Y_{l} \lambda(t)\right)(z)-\lambda(z)\right| \leq & \left(Y_{l}^{*}\left|\lambda(t)-\tilde{\lambda}_{s}(t)\right|\right)(z)+\left|\lambda(z)-\tilde{\lambda}_{s}(z)\right| \\
& +\left|\left(Y_{l}^{*} \tilde{\lambda}_{s}(t)\right)(z)-\tilde{\lambda}_{s}(z)\right| \\
& +\left|\lambda\left(z+A_{l}(z)\right)-\lambda(z)\right| .
\end{aligned}
$$

Combining (23) and (44), we have

$$
\begin{aligned}
w_{r}(z)\left|\left(Y_{l} \lambda(t)\right)(z)-\lambda(z)\right| \leq & \left(C_{r}+3\right)\left\|\lambda-\tilde{\lambda}_{s}\right\|_{r} \\
& +C_{r}\left\|\tilde{\lambda}_{s}^{\prime \prime}\right\|_{r} \frac{z^{2}}{l} w_{r}(z)\left|\lambda\left(z+A_{l}(z)\right)-\lambda(z)\right| \\
\leq & C_{r} \omega_{r}^{2}(\lambda ; s)\left(1+\frac{1}{s^{2}} \frac{z^{2}}{l}\right) \\
& +\omega_{r}^{1}\left(\lambda ; A_{l}(z)\right) .
\end{aligned}
$$

Hence, choosing $s=z / \sqrt{l}$, the first part of the proof is proved. The second part of the proof can be directly observed from the definition of the space $\operatorname{Lip}_{r}^{2} \alpha$.

\section{Direct Results}

\subsection{Voronovskaya-Type Theorem}

Theorem 12. If $\lambda \in C_{B}\left(\mathbb{R}_{+}\right)$and $\lambda^{\prime \prime}$ exists at a point $z \in \mathbb{R}_{+}$, then

$$
\lim _{l \longrightarrow \infty} l\left(\left(Y_{l} \lambda(t)\right)(z)-\lambda(z)\right)=3 z\left(\lambda^{\prime}(z)+z \lambda^{\prime \prime}(z)\right)
$$

Proof. By the Taylor's expansion formula for $\lambda$, we have

$$
\lambda(t)=\lambda(z)+\lambda^{\prime}(z)(t-z)+\frac{1}{2} \lambda^{\prime \prime}(z)(t-z)^{2}+R(t ; z)(t-z)^{2},
$$

where

$$
R(t ; z)= \begin{cases}\frac{\lambda(t)-\lambda(z)-\lambda^{\prime}(z)(t-z)-1 / 2 \lambda^{\prime \prime}(z)(t-z)^{2}}{(t-z)^{2}}, & t \neq z ; \\ 0, & t=z .\end{cases}
$$

Applying the L'Hospital's Rule,

$$
\lim _{t \rightarrow z} R(t ; z)=\frac{1}{2} \lim _{t \rightarrow z} \frac{\lambda^{\prime}(t)-\lambda^{\prime}(z)}{t-z}-\frac{1}{2} \lambda^{\prime \prime}(z)=0 .
$$

Thus, $R(. ; z) \in C_{B}\left(\mathbb{R}_{+}\right)$. Consequently, we can write

$$
\begin{aligned}
\left(Y_{l} \lambda(t)\right)(z)-\lambda(z)= & A_{l}(z) \lambda^{\prime}(z)+\frac{1}{2} B_{l}(z) \lambda^{\prime \prime}(z) \\
& +\left(Y_{l}\left(R(t ; z)(t-z)^{2}\right)\right)(z) .
\end{aligned}
$$

By the Cauchy-Schwarz inequality, we have $l\left(Y_{l}\left(R(t ; z)(t-z)^{2}\right)\right)(z) \leq \sqrt{\left(Y_{l}\left(R^{2}(t ; z)\right)\right)(z)} \sqrt{l^{2}\left(Y_{l}\left((t-z)^{4}\right)\right)(z)}$.

We observe that $R^{2}(z ; z)=0$ and $R^{2}(t ; z) \in C_{B}\left(\mathbb{R}_{+}\right)$. Then, it follows in Lemma 5 that 


$$
\lim _{l \longrightarrow \infty}\left(Y_{l}\left(R^{2}(t ; z)\right)\right)(z)=R^{2}(z ; z)=0 .
$$

Hence, from (17), we can obtain

$$
\lim _{l \longrightarrow \infty} l\left(Y_{l}\left(R(t ; z)(t-z)^{2}\right)\right)(z)=0 .
$$

Combining (15) and (16), we complete the proof of Theorem 12.

Corollary 13. If $\lambda, \lambda^{\prime \prime} \in C_{B}\left(\mathbb{R}_{+}\right)$, then we have

$$
\lim _{l \longrightarrow \infty} l\left(\left(Y_{l} \lambda(t)\right)(z)-\lambda(z)\right)=3 z\left(\lambda^{\prime}(z)+z \lambda^{\prime \prime}(z)\right)
$$

uniformly with respect to any finite interval $I \subset \mathbb{R}_{+}$.

4.2. Point-Wise Estimates. In this subsection, we establish three point-wise estimates of the operators (2). First, we obtain the rate of convergence locally by using functions belonging to the Lipschitz class. We denote that $\lambda \in C_{B}\left(\mathbb{R}_{+}\right)$ is in $\operatorname{Lip}_{M}(\gamma, D), \gamma \in(0,1]$, and $D \subset \mathbb{R}_{+}$if it satisfies the following condition:

$$
|\lambda(t)-\lambda(z)| \leq M|t-z|^{\gamma}, t \in D, z \in \mathbb{R}_{+},
$$

where $M$ is a positive constant depending only on $\gamma$ and $\lambda$.

Theorem 14. If $\lambda \in C_{B}\left(\mathbb{R}_{+}\right) \bigcap \operatorname{Lip}_{M}(\gamma, D)$, then for any $z \in$ $\mathbb{R}_{+}$, we have

$$
\left|\left(Y_{l} \lambda(t)\right)(z)-\lambda(z)\right| \leq M\left(\left(B_{l}(z)\right)^{\gamma / 2}+2 d(z ; D)\right)
$$

where $d(z ; D)=\inf \{|t-z|: t \in D\}$ denotes the distance between $z$ and $D$.

Proof. Let $\bar{D}$ be the closure of $D$. Using the properties of infimum, there is at least a point $t_{0} \in \bar{D}$ such that $\mathrm{d}(z ; D)=$ $\left|z-t_{0}\right|$. By the triangle inequality

$$
|\lambda(t)-\lambda(z)| \leq\left|\lambda(t)-\lambda\left(t_{0}\right)\right|+\left|\lambda(z)-\lambda\left(t_{0}\right)\right|
$$

we have

$$
\begin{aligned}
\left|\left(Y_{l} \lambda(t)\right)(z)-\lambda(z)\right| & \leq\left(Y_{l}\left|\lambda(t)-\lambda\left(t_{0}\right)\right|\right)(z)+\left(Y_{l}\left|\lambda(z)-\lambda\left(t_{0}\right)\right|\right)(z) \\
& \leq M\left\{\left(Y_{l}\left|t-t_{0}\right|^{\gamma}\right)(z)+\left|z-t_{0}\right|^{\gamma}\right\} \\
& \leq M\left\{\left(Y_{l}\left(|t-z|^{\gamma}+\left|z-t_{0}\right|^{\gamma}\right)\right)(z)+\left|z-t_{0}\right|^{\gamma}\right\} \\
& \leq M\left\{\left(Y_{l}|t-z|^{\gamma}\right)(z)+2\left|z-t_{0}\right|^{\gamma}\right\} .
\end{aligned}
$$

Choosing $p=2 / \gamma$ and $q=2 / 2-\gamma$ and using the well- known Hölder inequality, we have

$$
\begin{aligned}
& \left|\left(Y_{l} \lambda(t)\right)(z)-\lambda(z)\right| \\
& \quad \leq M\left\{\left(\left(Y_{l}|t-z|^{p \gamma}\right)(z)\right)^{1 / p}\left(\left(Y_{l} 1^{q}\right)(z)\right)^{1 / q}+2 \mathrm{~d}^{\gamma}(z ; D)\right\} \\
& \quad \leq M\left\{\left(\left(Y_{l}(t-z)^{2}\right)(z)\right)^{\gamma / 2}+2 \mathrm{~d}^{\gamma}(z ; D)\right\} \\
& \quad \leq M\left(\left(B_{l}(z)\right)^{\gamma / 2}+2 \mathrm{~d}^{\gamma}(z ; D)\right) .
\end{aligned}
$$

Next, we obtain the local direct estimate of the operators (2), using the Lipcshitz type maximal function of the order $\gamma$ introduced by Lenze [25] as

$$
\tilde{\omega}_{\gamma}(\lambda ; z)=\sup _{z, t \in \mathbb{R}_{+}, t \neq z} \frac{|\lambda(t)-\lambda(z)|}{|t-z|^{\gamma}}, \gamma \in(0,1] \text {. }
$$

Theorem 15. If $\lambda \in C_{B}\left(\mathbb{R}_{+}\right)$, then for any $z \in \mathbb{R}_{+}$, we have

$$
\left|\left(Y_{l} \lambda(t)\right)(z)-\lambda(z)\right| \leq \tilde{\omega}_{\gamma}(\lambda ; z)\left(B_{l}(z)\right)^{\gamma / 2}
$$

Proof. From equation (70), we have

$$
\left|\left(Y_{l} \lambda(t)\right)(z)-\lambda(z)\right| \leq \tilde{\omega}_{\gamma}(\lambda ; z)\left(Y_{l}(t-z)^{\gamma}\right)(z) .
$$

Applying the well-known Hölder inequality, we have

$$
\begin{aligned}
\left|\left(Y_{l} \lambda(t)\right)(z)-\lambda(z)\right| & \leq \tilde{\omega}_{\gamma}(\lambda ; z)\left(\left(Y_{l}(t-z)^{2}\right)(z)\right)^{\gamma / 2} \\
& =\tilde{\omega}_{\gamma}(\lambda ; z)\left(B_{l}(z)\right)^{\gamma / 2} .
\end{aligned}
$$

Finally, we establish point-wise estimate of the operators (2) in the following Lipschitz-type space (see [26]) with two distinct parameters $\mu_{1}, \mu_{2} \in \mathbb{R}_{+}$:

$\operatorname{Lip}_{M}^{\left(\mu_{1}, \mu_{2}\right)}(\gamma):=\left\{\lambda \in C\left(\mathbb{R}_{+}\right):|\lambda(t)-\lambda(z)| \leq M \frac{|t-z|^{\gamma}}{t+\mu_{1} z^{2}+\mu_{2} z}\right\}, t, z \in \mathbb{R}_{+}$,

where $\gamma \in(0,1], M$, is a positive constant depending only on $\gamma, \mu_{1}, \mu_{2}$ and $\lambda$.

Theorem 16. If $\lambda \in \operatorname{Lip}_{M}^{\left(\mu_{1}, \mu_{2}\right)}(\gamma)$, then for any $z \in \mathbb{R}_{+}$, we have

$$
\left|\left(Y_{l} \lambda(t)\right)(z)-\lambda(z)\right| \leq M\left(\frac{B_{l}(z)}{\mu_{1} z^{2}+\mu_{2} z}\right)^{\gamma / 2}
$$

Proof. Applying the well-known Hölder inequality with $p=2 / \gamma$ and $q=2 / 2-\gamma$, we have 


$$
\begin{aligned}
\left|\left(Y_{l} \lambda(t)\right)(z)-\lambda(z)\right| & \leq\left(Y_{l}|\lambda(t)-\lambda(z)|\right)(z) \\
& \leq\left(Y_{l} M \frac{|t-z|^{\gamma}}{t+\mu_{1} z^{2}+\mu_{2} z}\right)(z) \\
& \leq \frac{M}{\mu_{1} z^{2}+\mu_{2} z}\left(Y_{l}|t-z|^{\gamma}\right)(z) \\
& \leq \frac{M}{\mu_{1} z^{2}+\mu_{2} z}\left(\left(Y_{l}|t-z|^{p \gamma}\right)(z)\right)^{1 / p}\left(\left(Y_{l} 1^{q}\right)(z)\right)^{1 / q} \\
& =M\left(\frac{B_{l}(z)}{\mu_{1} z^{2}+\mu_{2} z}\right)^{\gamma / 2} .
\end{aligned}
$$

Thus, the proof is completed.

\section{Weighted Approximation}

Let $B_{2}\left(\mathbb{R}_{+}\right)$be the set of all functions $\lambda$ defined on $\mathbb{R}_{+}$satisfying the condition $|\lambda(z)| \leq M_{\lambda}\left(1+z^{2}\right)$ with an absolute constant $M_{\lambda}>0$ which depends only on $\lambda . C_{2}\left(\mathbb{R}_{+}\right)$denotes the subspace of all continuous functions $\lambda \in B_{2}\left(\mathbb{R}_{+}\right)$with the norm $\|\lambda\|_{2}=\sup _{z \in \mathbb{R}_{+}}\left(|\lambda(z)| / 1+z^{2}\right)$. By $C_{2}^{0}\left(\mathbb{R}_{+}\right)$, we denote the subspace of all functions $f \in C_{2}\left(\mathbb{R}_{+}\right)$for which $\lim _{z \longrightarrow+\infty}|\lambda(z)|$ $/ 1+z^{2}$ is finite.

Theorem 17. If $\lambda \in C_{2}^{0}\left(\mathbb{R}_{+}\right)$and $\kappa>0$, we have

$$
\lim _{l \longrightarrow \infty} \sup _{z \in \mathbb{R}_{+}} \frac{\left|\left(Y_{l} \lambda(t)\right)(z)-\lambda(z)\right|}{\left(1+z^{2}\right)^{1+\kappa}}=0 .
$$

Proof. Let $z_{0} \in \mathbb{R}_{+}$be arbitrary but fixed.

$$
\begin{aligned}
\sup _{z \in \mathbb{R}_{+}} \frac{\left|\left(Y_{l} \lambda(t)\right)(z)-\lambda(z)\right|}{\left(1+z^{2}\right)^{1+\kappa}} \leq \sup _{z \in\left(0, z_{0}\right)} \frac{\left|\left(Y_{l} \lambda(t)\right)(z)-\lambda(z)\right|}{\left(1+z^{2}\right)^{1+\kappa}} & \\
& +\sup _{z \in\left[z_{0}, \infty\right)} \frac{\left|\left(Y_{l} \lambda(t)\right)(z)-\lambda(z)\right|}{\left(1+z^{2}\right)^{1+\kappa}} \\
\leq & \left\|\left(Y_{l} \lambda(t)\right)(z)-\lambda\right\|_{\left(0, z_{0}\right)} \\
& +\|\lambda\|_{2} \sup _{z \in\left[z_{0}, \infty\right)} \frac{\left(Y_{l}\left(1+t^{2}\right)\right)(z)}{\left(1+z^{2}\right)^{1+\kappa}} \\
& +\sup _{z \in\left[z_{0}, \infty\right)} \frac{|\lambda(z)|}{\left(1+z^{2}\right)^{1+\kappa}}:=I_{1}+I_{2}+I_{3} .
\end{aligned}
$$

Applying $|\lambda(z)| \leq\|\lambda\|_{2}\left(1+z^{2}\right)$, we have

$$
I_{3}=\sup _{z \in\left[z_{0}, \infty\right)} \frac{|\lambda(z)|}{\left(1+z^{2}\right)^{1+\kappa}} \leq \sup _{z \in\left[z_{0}, \infty\right)} \frac{\|\lambda\|_{2}\left(1+z^{2}\right)}{\left(1+z^{2}\right)^{1+\kappa}} \leq \frac{\|\lambda\|_{2}}{\left(1+z_{0}^{2}\right)^{\kappa}} .
$$

Let $\varepsilon>0$. Since $\lim _{l \longrightarrow \infty} \sup _{z \in\left[z_{0}, \infty\right)}\left(Y_{l}\left(1+t^{2}\right)\right)(z) /\left(1+z^{2}\right)=1$, there exists $L_{1} \in \mathbb{N}$, such that for all $l>L_{1}$,

$$
\begin{aligned}
\frac{\|\lambda\|_{2}\left(Y_{l}\left(1+t^{2}\right)\right)(z)}{\left(1+z^{2}\right)^{1+\kappa}} & \leq \frac{\|\lambda\|_{2}}{\left(1+z^{2}\right)^{1+\kappa}}\left(\left(1+z^{2}\right)+\frac{\varepsilon}{3\|\lambda\|_{2}}\right) \\
& \leq \frac{\|\lambda\|_{2}}{\left(1+z^{2}\right)^{\kappa}}+\frac{\varepsilon}{3} .
\end{aligned}
$$

Hence,

$$
\|\lambda\|_{2} \sup _{z \in\left[z_{0}, \infty\right)} \frac{\left(Y_{l}\left(1+t^{2}\right)\right)(z)}{\left(1+z^{2}\right)^{1+\kappa}} \leq \frac{\|\lambda\|_{2}}{\left(1+z_{0}^{2}\right)^{\kappa}}+\frac{\varepsilon}{3}, \forall l \geq L_{1} .
$$

Thus,

$$
I_{2}+I_{3}<\frac{2\|\lambda\|_{2}}{\left(1+z_{0}^{2}\right)^{\kappa}}+\frac{\varepsilon}{3}, \forall l \geq L_{1}
$$

Next, for sufficiently large $z_{0}$ such that $\|\lambda\|_{2} /\left(1+z_{0}^{2}\right)^{\kappa}<$ $\varepsilon / 6$, then $I_{2}+I_{3}<2 \varepsilon / 3, \forall l \geq L_{1}$. Applying Lemma 5, there exists $L_{2} \in \mathbb{N}$, such that for all $l>L_{2}$,

$$
\left\|\left(Y_{l} \lambda(t)\right)(z)-\lambda\right\|_{\left(0, z_{0}\right)}<\frac{\varepsilon}{3}
$$
have

Let $L=\max \left\{L_{1}, L_{2}\right\}$. Combining (80) (82), and (83), we

$$
\sup _{z \in \mathbb{R}_{+}} \frac{\left|\left(Y_{l} \lambda(t)\right)(z)-\lambda(z)\right|}{\left(1+z^{2}\right)^{1+\kappa}}<\varepsilon, \forall l \geq L
$$

Hence, the proof of Theorem 17 is completed.

Theorem 18. If $\lambda \in C_{2}^{0}\left(\mathbb{R}_{+}\right)$, then we have

$$
\lim _{l \longrightarrow \infty}\left\|\left(Y_{l} \lambda(t)\right)(z)-\lambda\right\|_{2}=0
$$

Proof. Applying the Korovkin theorem [27], it is sufficient to show the following three conditions:

$$
\lim _{l \longrightarrow \infty}\left\|\left(Y_{l} t^{p}\right)(z)-z^{p}\right\|_{2}=0, p=0,1,2
$$

Since $\left(Y_{l} 1\right)(z)=1$, the condition (86) holds for $p=0$. From Lemma (11), we have

$$
\left\|\left(Y_{l} t\right)(z)-z\right\|_{2}=\sup _{z \in \mathbb{R}_{+}} \frac{1}{1+z^{2}}\left|\frac{l+3}{l} z-z\right| \leq \frac{3}{l}
$$

Thus, $\lim _{l \longrightarrow \infty}\left\|\left(Y_{l} t\right)(z)-z\right\|_{2}=0$. Finally, we have

$$
\left\|\left(Y_{l} t^{2}\right)(z)-z^{2}\right\|_{2}=\sup _{z \in \mathbb{R}_{+}} \frac{1}{1+z^{2}}\left|\frac{(l+2)(l+9)}{l(l-1)} z^{2}-z^{2}\right| \leq \frac{12 l+19}{l(l-1)},
$$

which implies that $\lim _{l \longrightarrow \infty}\left\|\left(Y_{l} t^{2}\right)(z)-z^{2}\right\|_{2}=0$. 


\section{Some Voronovskaya-Type Approximation Theorem}

As is known, if $\lambda \in C\left(\mathbb{R}_{+}\right)$is not uniform, the limit $\lim _{\delta \rightarrow 0^{+}} \omega(\lambda$; $\delta)=0$ may be not true. In [28], Yüksel and Ispir defined the following weighted modulus of continuity:

$$
\Omega(\lambda ; \delta)=\sup _{z \in \mathbb{R}_{+}, 0<h \leq \delta} \frac{|\lambda(z+h)-\lambda(z)|}{\left(1+z^{2}\right)\left(1+h^{2}\right)} \text { for } \lambda \in C_{2}^{0}\left(\mathbb{R}_{+}\right)
$$

and proved the properties of monotone increasing about $\Omega(\lambda ; \delta)$ as $\delta>0, \lim _{\delta \longrightarrow 0^{+}} \Omega(\lambda ; \delta)=0$, and the inequality

$$
\Omega(\lambda ; \tau \delta) \leq 2(1+\tau)\left(1+\delta^{2}\right) \Omega(\lambda ; \delta), \tau>0
$$

For any $\lambda \in C_{2}^{0}\left(\mathbb{R}_{+}\right)$, it follows from (89) and (90) that

$$
\begin{aligned}
|\lambda(t)-\lambda(z)| & \leq\left(1+(t-z)^{2}\right)\left(1+z^{2}\right) \Omega(\lambda ;|t-z|) \\
& \leq 2\left(1+\frac{|t-z|}{\delta}\right)\left(1+\delta^{2}\right) \Omega(\lambda ; \delta)\left(1+(t-z)^{2}\right)\left(1+z^{2}\right) .
\end{aligned}
$$

In the next theorem, we obtain the degree of approximation of $\lambda$ by the operators (2) in the weighted space of continuous functions $C_{2}^{0}\left(\mathbb{R}_{+}\right)$in terms of the weighted modulus of smoothness $\Omega(\lambda ; \delta), \delta>0$.

\subsection{Quantitative Voronovskaya-Type Theorem}

Theorem 19. If $\lambda \in C_{2}^{0}\left(\mathbb{R}_{+}\right)$satisfies $\lambda^{\prime}, \lambda^{\prime \prime} \in C_{2}^{0}\left(\mathbb{R}_{+}\right)$, then for sufficiently large $l$ and any $z \in \mathbb{R}_{+}$,

$$
l\left|\left(Y_{l} \lambda(t)\right)(z)-\lambda(z)-\lambda^{\prime}(z) A_{l}(z)-\frac{\lambda^{\prime \prime}(z)}{2 !} B_{l}(z)\right| \leq O(1) \Omega\left(\lambda^{\prime \prime} ; \frac{1}{\sqrt{l}}\right) .
$$

Proof. By Taylors' expansion formula for $\lambda$, we have

$$
\begin{aligned}
\lambda(t) & =\lambda(z)+\lambda^{\prime}(z)(t-z)+\frac{\lambda^{\prime \prime}(y)}{2 !}(t-z)^{2} \\
& =\lambda(z)+\lambda^{\prime}(z)(t-z)+\frac{\lambda^{\prime \prime}(z)}{2 !}(t-z)^{2}+R_{1}(t, z),
\end{aligned}
$$

where $|y-z| \leq|t-z|$ and hence

$$
R_{1}(t, z)=\frac{\lambda^{\prime \prime}(y)-\lambda^{\prime \prime}(z)}{2 !}(t-z)^{2}
$$

Applying the inequality (91) of the weighted modulus of continuity, we have

$$
\begin{aligned}
& \left|\lambda^{\prime \prime}(y)-\lambda^{\prime \prime}(z)\right| \leq\left(1+(y-z)^{2}\right)\left(1+z^{2}\right) \Omega\left(\lambda^{\prime \prime} ;|y-z|\right) \\
& \leq\left(1+(t-z)^{2}\right)\left(1+z^{2}\right) \Omega\left(\lambda^{\prime \prime} ;|t-z|\right) \\
& \leq 2\left(1+\frac{|t-z|}{\delta}\right)\left(1+\delta^{2}\right) \Omega\left(\lambda^{\prime \prime} ; \delta\right) \\
& \cdot\left(1+(t-z)^{2}\right)\left(1+z^{2}\right) \\
& \leq\left\{\begin{array}{l}
4\left(1+\delta^{2}\right)^{2}\left(1+z^{2}\right) \Omega\left(\lambda^{\prime \prime} ; \delta\right),|t-z| \leq \delta, \\
4\left(1+\delta^{2}\right)^{2}\left(1+z^{2}\right) \Omega\left(\lambda^{\prime \prime} ; \delta\right) \frac{(t-z)^{4}}{\delta^{4}},|t-z|>\delta,
\end{array}\right. \\
& \leq 4\left(1+\delta^{2}\right)^{2}\left(1+z^{2}\right) \Omega\left(\lambda^{\prime \prime} ; \delta\right)\left(1+\frac{(t-z)^{4}}{\delta^{4}}\right) \text {. }
\end{aligned}
$$

Combining (94) and (95) and choosing $\delta \in(0,1)$, we have

$$
\left|R_{1}(t, z)\right| \leq 2\left(1+\delta^{2}\right)^{2}\left(1+z^{2}\right) \Omega\left(\lambda^{\prime \prime} ; \delta\right)\left(1+\frac{(t-z)^{4}}{\delta^{4}}\right)(t-z)^{2} .
$$

Using the operator (2) and Lemma 4 on both sides of (94), we have

$$
\begin{aligned}
& \left|\left(Y_{l} \lambda(t)\right)(z)-\lambda(z)-\lambda^{\prime}(z) A_{l}(z)-\frac{\lambda^{\prime \prime}(z)}{2 !} B_{l}(z)\right| \\
& \quad \leq\left(Y_{l}\left|R_{1}(t, z)\right|\right)(z) .
\end{aligned}
$$

Applying (16), (18), and (96), we have

$$
\begin{aligned}
\left(Y_{l}\left|R_{1}(t, z)\right|\right)(z) \leq & 2\left(1+\delta^{2}\right)^{2}\left(1+z^{2}\right) \Omega\left(\lambda^{\prime \prime} ; \delta\right) \\
& \cdot\left(Y_{l}\left((t-z)^{2}+\frac{(t-z)^{6}}{\delta^{4}}\right)\right)(z) \\
\leq & 2\left(1+\delta^{2}\right)^{2}\left(1+z^{2}\right) \Omega\left(\lambda^{\prime \prime} ; \delta\right) \\
& \cdot\left(B_{l}(z)+\frac{1}{\delta^{4}}\left(Y_{l}(t-z)^{6}\right)(z)\right) \\
\leq & 2\left(1+\delta^{2}\right)^{2}\left(1+z^{2}\right) \Omega\left(\lambda^{\prime \prime} ; \delta\right) \\
& \cdot\left(O\left(\frac{1}{l}\right)+\frac{1}{\delta^{4}} O\left(\frac{1}{l^{3}}\right)\right) .
\end{aligned}
$$

Choosing $\delta=1 / \sqrt{l}$, we have

$$
l\left(Y_{l}\left|R_{1}(t, z)\right|\right)(z) \leq O(1) \Omega\left(\lambda^{\prime \prime} ; \frac{1}{\sqrt{l}}\right) .
$$

Combining (97)-(99), we complete the proof of Theorem 19.

6.2. Grüss Voronovskaya-Type Theorem 
Theorem 20. If $\lambda, \mu \in C_{2}^{0}\left(\mathbb{R}_{+}\right)$satisfy $\lambda \mu, \lambda^{\prime}, \mu^{\prime},(\lambda \mu)^{\prime}, \lambda^{\prime \prime}, \mu^{\prime \prime}$ and $(\lambda \mu)^{\prime \prime} \in C_{2}^{0}\left(\mathbb{R}_{+}\right)$. Then, for any $z \in \mathbb{R}_{+}$,

$\lim _{l \longrightarrow \infty} l\left(\left(Y_{l}(\lambda \cdot \mu)(t)\right)(z)-\left(Y_{l} \lambda(t)\right)(z) \cdot\left(Y_{l} \mu(t)\right)(z)\right)=6 \lambda^{\prime}(z) \mu^{\prime}(z) z^{2}$.

Proof. Using the equalities

$$
\begin{aligned}
(\lambda \cdot \mu)(z) & =\lambda(z) \cdot \mu(z),(\lambda \cdot \mu)^{\prime}(z)=\lambda^{\prime}(z) \cdot \mu(z)+\lambda(z) \cdot \mu^{\prime}(z), \\
(\lambda \cdot \mu)^{\prime \prime}(z) & =\lambda^{\prime \prime}(z) \cdot \mu(z)+2 \lambda^{\prime}(z) \cdot \mu^{\prime}(z)+\lambda(z) \cdot \mu^{\prime \prime}(z),
\end{aligned}
$$

by simple computations, for any $z \in \mathbb{R}_{+}$, we have

$$
\begin{aligned}
&\left(Y_{l}(\lambda \cdot \mu)(t)\right)(z)-\left(Y_{l} \lambda(t)\right)(z) \cdot\left(Y_{l} \mu(t)\right)(z) \\
&=\left\{\left(Y_{l}(\lambda \cdot \mu)(t)\right)(z)-(\lambda \cdot \mu)(z)-(\lambda \cdot \mu)^{\prime}(z) A_{l}(z)-\frac{(\lambda \cdot \mu)^{\prime \prime}(z)}{2 !} B_{l}(z)\right\} \\
&-\mu(z)\left\{\left(Y_{l} \lambda(t)\right)(z)-\lambda(z)-\lambda^{\prime}(z) A_{l}(z)-\frac{\lambda^{\prime \prime}(z)}{2 !} B_{l}(z)\right\} \\
&-\left(Y_{l} \lambda(t)\right)(z)\left\{\left(Y_{l} \mu(t)\right)(z)-\mu(z)-\mu^{\prime}(z) A_{l}(z)-\frac{\mu^{\prime \prime}(z)}{2 !} B_{l}(z)\right\} \\
&+\frac{1}{2 !} B_{l}(z)\left\{\lambda(z) \cdot \mu^{\prime \prime}(z)+2 \lambda^{\prime}(z) \cdot \mu^{\prime}(z)-\mu^{\prime \prime}(z) \cdot\left(Y_{l} \lambda(t)\right)(z)\right\} \\
&+A_{l}(z)\left\{\lambda(z) \cdot \mu^{\prime}(z)-\mu^{\prime}(z) \cdot\left(Y_{l} \lambda(t)\right)(z)\right\} .
\end{aligned}
$$

By using (16), Lemma 5, and Theorem 19, we have

$\lim _{l \longrightarrow \infty} l\left(\left(Y_{l}(\lambda \cdot \mu)(t)\right)(z)-\left(Y_{l} \lambda(t)\right)(z) \cdot\left(Y_{l} \mu(t)\right)(z)\right)=6 \lambda^{\prime}(z) \mu^{\prime}(z) z^{2}$

which proves our theorem.

\section{Data Availability}

No data were used to support this study.

\section{Conflicts of Interest}

The authors declare that they have no conflicts of interest.

\section{Acknowledgments}

This work is supported by the National Natural Science Foundation of China (Grant Nos. 11626031, 11501143, and 61962011), the Key Natural Science Research Project in Universities of Anhui Province (Grant No. KJ2019A0572), the Philosophy and Social Sciences General Planning Project of Anhui Province of China (Grant No. AHSKYG2017D153), the Natural Science Foundation of Anhui Province of China (Grant No. 1908085QA29), the Guizhou Provincial Science and Technology Foundation under Grant [2019]1434, and the Doctoral Research Project of Guizhou Normal University (No. GZNUD [2017]27).

\section{References}

[1] H. Karsli, "Rate of convergence of new Gamma type operators for functions with derivatives of bounded variation," Mathematical and Computer Modelling, vol. 45, no. 5-6, pp. 617-624, 2007.

[2] A. İzgi and İ. Buyukyazici, "Approximation in boundedness interval and order of approximation," Kastamonu Eğitim Dergisi, vol. 11, no. 2, pp. 451-460, 2003.

[3] H. Karsli, V. Gupta, and A. İzgi, "Rate of pointwise convergence of a new kind of gamma operators for functions of bounded variation," Applied Mathematics Letters, vol. 22, pp. 505-510, 2009.

[4] H. Karsli and M. A. Özarslan, "Direct local and global approximation results for operators of gamma type," Hacettepe Journal of Mathematics and Statistics, vol. 39, pp. 241-253, 2010.

[5] A. İzgi, "Voronovskaya type asymptotic approximation by modified Gamma operators," Applied Mathematics and Computation, vol. 217, no. 20, pp. 8061-8067, 2011.

[6] G. Krech, "A note on the paper "Voronovskaya type asymptotic approximation by modified gamma operators"," Applied Mathematics and Computation, vol. 219, no. 11, pp. 57875791, 2013

[7] G. Krech, "On the rate of convergence for modified gamma operators," Revista De La Unión Mathmátuca Argentina, vol. 55, no. 2, pp. 123-131, 2014.

[8] Q. B. Cai and X. M. Zeng, "On the convergence of a kind of q-gamma operators," Journal of Inequalities and Applications, vol. 2013, no. 1, 2013.

[9] C. Zhao, W. T. Cheng, and X. M. Zeng, "Some approximation properties of a kind of $q$-Gamma Stancu," Journal of Inequalities and Applications, vol. 94, 2014.

[10] W. T. Cheng, W. H. Zhang, and Q. B. Cai, “( $p, q)$-gamma operators which preserve $x^{2}$, "Journal of Inequalities and Applications, vol. 2019, 2019.

[11] X. L. Zhou, W. T. Cheng, and Q. B. Cai, "On Stancu type generalization of $(p, q)$-Gamma operators," Journal of Nonlinear Functional Analysis, vol. 2020, p. 12, 2020.

[12] T. Acar, M. Mursaleen, and S. N. Deveci, "Gamma operators reproducing exponential functions," Advances in Difference Equations, vol. 2020, no. 1, 2020.

[13] W. T. Cheng and Q. B. Cai, "Generalized ( $p, q)$-Gamma-type operators," Journal of Functions Spaces, vol. 2020, article 8978121, 10 pages, 2020.

[14] W. T. Cheng and X. J. Tang, "Approximation properties of $\lambda$-Gamma operators based on $q$-integers," Journal of Functions Spaces, vol. 2020, article 5710510, 2020.

[15] W. T. Cheng and W. H. Zhang, "On $(p, q)$-analogue of Gamma operators," Journal of Functions Spaces, vol. 2019, article 9607517, 2019.

[16] W. T. Cheng, W. H. Zhang, and J. Zhang, “Approximation properties of modified $q$-gamma operators preseving linear functions," Filomat, vol. 34, no. 5, pp. 1601-1609, 2020.

[17] S. N. Deveci, T. Acar, and O. Alagöz, "Approximation by gamma type operators," Mathematical Methods in the Applied Sciences, vol. 43, no. 5, pp. 2772-2782, 2020.

[18] T. Acar, "Asymptotic formulas for generalized Szász-Mirakyan operators," Applied Mathematics and Computation, vol. 263, pp. 233-239, 2015.

[19] T. Acar, "Quantitative $q$-Voronovskaya and $q$-GrüssVoronovskaya-type results for q-Szász operators," Georgian Mathematical Journal, vol. 23, no. 4, pp. 459-468, 2016. 
[20] T. Acar, A. Aral, and I. Rasa, "The new forms of Voronovskaya's theorem in weighted spaces," Positivity, vol. 20, no. 1, pp. 25-40, 2016.

[21] T. Acar and G. Ulusoy, "Approximation by modified SzászDurrmeyer operators," Periodica Mathematica Hungarica, vol. 72, no. 1, pp. 64-75, 2016.

[22] J. Bustamante and L. Flores de Jesús, "Quantitative Voronovskaya-type theorems for Fejér-Korovkin operators," Constructive Mathematical Analysis, vol. 3, no. 4, pp. 150164, 2020.

[23] J. Bustamante and L. Flores de Jesús, "Strong converse inequalities and qantitative Voronovskaya-type theorems for trigonometric Fejér sums," Constructive Mathematical Analysis, vol. 3, no. 2, pp. 53-63, 2020.

[24] G. Ulusoy and T. Acar, " $q$-Voronovskaya type theorems for qBaskakov operators," Mathematical Methods in the Applied Sciences, vol. 39, no. 12, pp. 3391-3401, 2016.

[25] B. Lenze, "On Lipschitz type maximal functions and their smoothness spaces," Indagationes Mathematicae, vol. 90, no. 1, pp. 53-63, 1988.

[26] M. A. Özarslan and H. Aktuğlu, "Local approximation for certain King-type operator," Filomat, vol. 27, no. 1, pp. 173-181, 2013.

[27] A. D. Gadzhiev, "Theorems of the type of P.P. Korovkin theorems," Matematicheskie Zametki, vol. 20, no. 5, pp. 781-786, 1976.

[28] I. Yüksel and N. Ispir, "Weighted approximation by a certain family of summation integral-type operators," Computers \& Mathematcs with Applications, vol. 52, no. 10-11, pp. 1463$1470,2006$. 